\title{
TESTING WATER POLLUTION BASED ON WIRELESS SENSOR NETWORKS AND STOCHASTIC APPROXIMATION METHOD: THE CASE OF FLINT, MICHIGAN, USA
}

\author{
NAHED A. ALNAHASH \& MOHAMED A. ZOHDY \\ School of Engineering and Computer Science, Oakland University, Rochester, USA
}

\begin{abstract}
Because water is essential to human life and a healthy environment, it is important that the water is in good condition to use. Water pollution is one of the important issues that affect the environment, so it should be monitored over time. A conventional method requires personnel to physically go to the location and take the samples from different places and bring them back to the lab to process. A novel monitoring system architecture based on a wireless sensor network was proposed in this research. As the use of this technology, it is important to have a good system to provide good analysis in a short time, cost, and efficiency. In this research, a wireless network was built using MatLab software, and the low-energy adaptive clustering hierarchy (LEACH) is considered the best algorithm in the construction of clusters. This research focuses on Flint water crisis. Stochastic approximation method is considered to calculate the collected sensors data and compare it with real data that was collected from Michigan.gov website. The lead rate is the element that this research focused on. Because this research using, wireless sensor networks the measurements will be mixed with noise at the base station, and the linear Kalman filter was applied to mitigating each sensor measurement noise. The simulation results showed that the surveillance system succeeded in calculating the data compared to the values from the US Environmental Protection Agency (EPA).

Keywords: LEACH, cluster head, sensors, WSN, stochastic method, lead, pollution, WHO, LKF.
\end{abstract}

\section{INTRODUCTION}

If we look at this land, we find that $71 \%$ of water covered the surface. This percentage is considered three-quarters of the area and any change on it such as a pollution which affects the humans, plants, and animals. The environmental pollution is the most serious disaster faced by human beings, pollution means the deterioration of the environment due to a defect in the compatibility of the elements that make them lose their ability to perform their natural role. Especially in the natural disposal of pollutants by natural processes in the environment of air, water and land. These days, scientists had used the traditional method that has taken more time, more effort and expensive in its cost.

As water monitoring system can be used to track water quality over time we should have a good system to do this in a short period of time, that gives reliable data and has a low cost of production.

From the late 2000s, new sensors were developed to detect different water quality parameters. Such as sensor to utilize biosensors, microelectronic mechanical systems, laser technology, etc. [1].

Wireless sensor network (WSN) is a special kind of wireless networking, consisting of small and especially distributed devices (nodes) which monitor and control physical environments from a remote location with better accuracy [2]. There are more different applications for measuring environmental monitoring, transportation and logistics, emergency response, Industrial applications, and more. One of the environmental monitors that focusing on in this research is water quality. Each node in wireless sensor networks 
consists of a battery, as an energy source, radio transceiver, and a small microcontroller. The system gathers some of the predetermined water parameters, like lead, copper, etc., depending on the zone of deployments such as sanitation, or industrial zones. This research focused on measure lead rate on water and comparing it with the maximum contaminant level (MCL) that the Environmental Protection Agency (EPA) state it to figure out which is the dangers areas of water, where the 15 parts per billion (ppb) of lead is listed as the action level [3], [4].

This paper is organized as follows: Section 2 presents the description of the problem, Section 3 briefly describes the related works. Section 4 overviews the network design of our system. Section 5 reports the methods and results. Finally, we conclude the paper with future works in Section 6.

\section{DESCRIPTION OF THE PROBLEM}

The Flint River is 78.3 miles long, and it drains 1,332 square miles of Michigan, in Lapeer, Genesee, Shiawassee, Saginaw, Oakland, Tuscola, and Sanilac counties. In this section, we are going to give a brief discussion about what happened in 2014 in Flint Michigan. When the drinking water source for the city of Flint was changed from Lake Huron and the Detroit River to the Flint River, the water crisis began. The water in the Flint River has high levels of chlorides that making it highly corrosive to lead pipes [5]. According to a study conducted by Virginia Tech professor Marc Edwards, a leading authority on water quality showed that the Flint River water destroys lead pipes at 19 times the rate of water piped from Detroit [5]. The chlorides do not affect a direct threat to fish, wildlife, or humans, but cause serious problems because they corrode lead and other metals in piping and plumbing [6].

Due to incomplete water treatment, over 100,000 residents were potentially exposed to high levels of lead in the drinking water. Several studies proved that lead contamination was present in the water supply. In 2015, Dr. Marc Edwards from Virginia Tech received a call from Flint resident to make a water test in their lab and in the following year, in January 2016, an emergency was declared from a federal state and the Flint residents were instructed to use only bottled or filtered water for uses.

The lab follows a conventional method of the test, and this method requires personnel to physically go to the Flint city and take the samples from different places and bring them back to the lab to process. This kind of methods will be costly, need more effort and the result may be not reliable. So, using new technology such as wireless sensor networks (WSN) will solve these problems [7].

\section{RELATED WORK}

Because water is essential to human life and a healthy environment, it is important that the water is in good condition to use. According to World Health Organization (WHO), around 842,000 people are estimated to die each year from diarrhoea because of unsafe drinking water, sewage, and hand-dirtiness [8]. Water pollution is one of the important issues that affect the environment, so it should be monitored over time. Nowadays, as the wireless technology getting more mature, the applications of the wireless system can be implemented in many different fields such as transportation and logistics, Industrial, environment monitoring, precision agriculture and animal tracking, health monitoring, security and surveillance, space exploration and smart home.

According to Hayat et al. [9] "Review of Cluster-based Energy Routing Protocols for WSNs" presented various hierarchical clustered based routing protocols of the WSN in the literature and explains its benefits. We figured that the LEACH protocol is the best protocol for clustering sensors based on the strengths of the LEACH to build our architecture. 
In the paper "Studying Water Quality Monitoring System Based on Wireless Sensor Networks (WSNs)" presented by Alnahash and Zohdy [10] the authors studied the quality of the water based on three different parameters $\mathrm{pH}$, Turbidity, and dioxide oxygen (DO) and stochastic approximation method is considered to calculate the collected sensors data and compare it with Word Health Organization rates for safe drinking water. We applied the same method but on the lead element only that this research focused on. Because what we figured from the previous research, wireless sensor networks the measurements will be mixed with noise at the base station, the linear Kalman filter was applied in this research to mitigating each sensor measurement noise.

\section{SYSTEM DESIGN AND DISTRIBUTION}

Assumed that the system is made up of several sensors and base station (BS) and they will distribute the sensors homogeneously. The WSN system is equipped with sensors and microcontroller units, receivers, power supplies and radio frequency (RF) transceivers. The sensor unit consists of several kinds of sensors to distribute in the water to detect the lead rate.

The sensor nods divided into two categorize: cluster head $(\mathrm{CH})$ and sensor node (SN). Cluster heads are responsible for transmits the data when they receive it from sensor nods to a base station (BS). While the sensor nods responsible for sensing and transmitting the data to the cluster heads. after that, the end users, who have the rights to access the base station, should do the last step to get the result.

\section{METHODS}

For this study, architecture of the wireless network was built using MatLab software, and the low-energy adaptive clustering hierarchy $(\mathrm{LEACH})$ is considered the algorithm to use for constructing the clusters. This research focuses on one approach: water pollution, using Flint water crisis, as a case of study. The node data measurements are based on different distributions methods (normal distribution, and Weibull distribution) to simulating lead in Flint River. Because this research used a WSN, the measurements are mixed with noise at

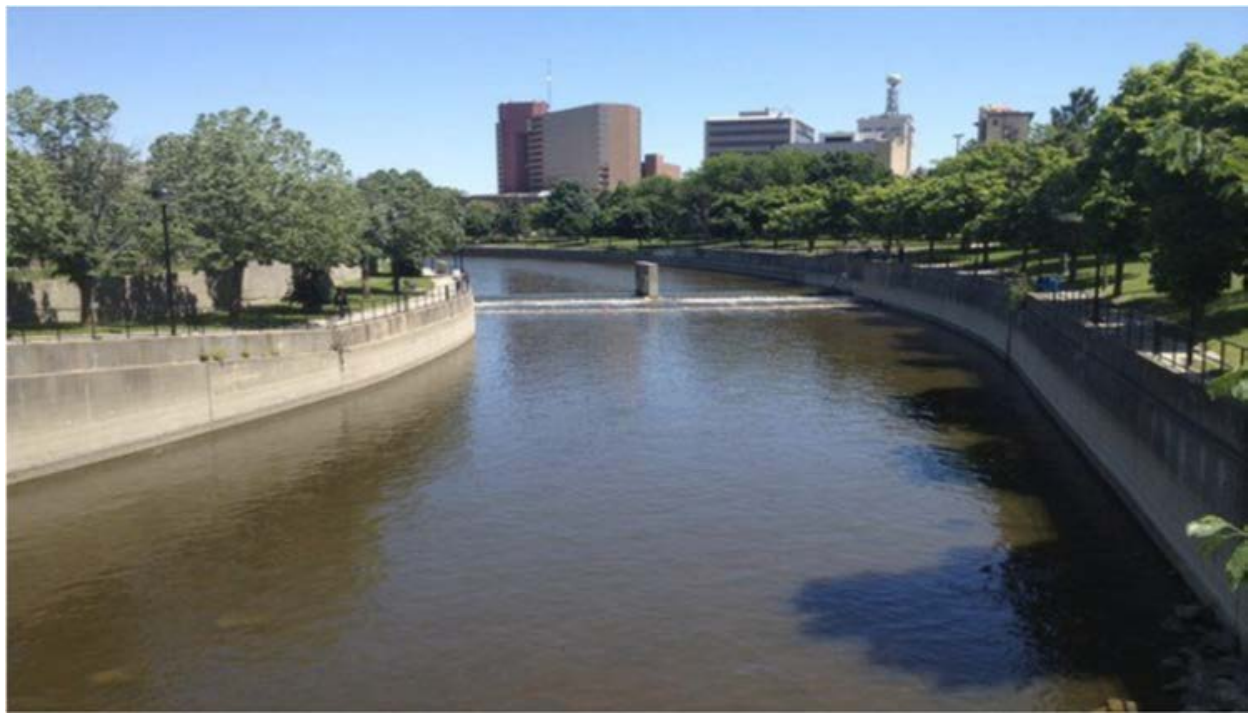

Figure 1: Flint River [11]. 


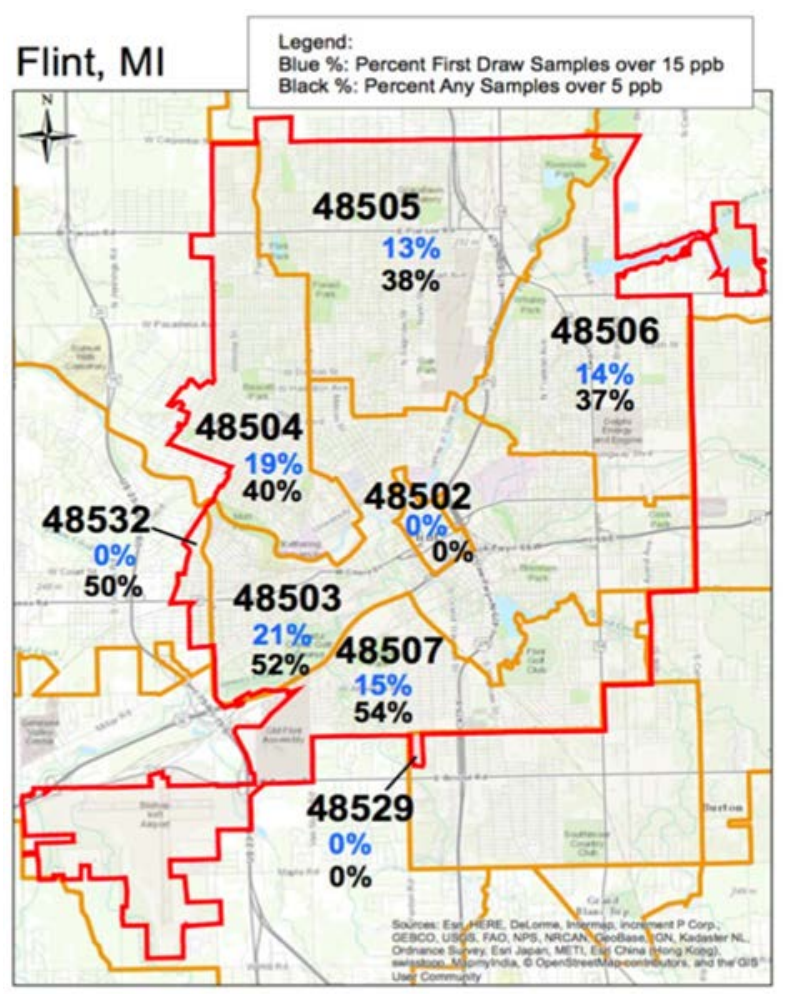

Figure 2: Map of the pilot area in Flint water crisis [12].

the BS. When the base station sends the data to the end user the simulation will be done by using a MatLab software and the output would look like Fig. 3(a) and (b), the linear Kalman filter is applied on the output to mitigate each sensor's measurement noise.

\section{SIMULATION RESULTS AND DISCUSSIONS}

This research has examined a scenario of water pollution. By using 100 sensors deployed randomly according to LEACH routing protocol across an area of $200 \mathrm{~m} \times 200 \mathrm{~m}$. Fig. 3(a) and (b) show the scenario output. This scenario focused on rate of lead on the water. Also, the result is observed from figures that all measurements have a noise and for this reason, these measurements need to be filtered via linear Kalman filter. Furthermore, Table 1 shows the studying result side-by-side with some real values [13]. All values measured as parts per billion (ppb), according to US Environmental Protection Agency (EPA) the water with 0 to less than $15 \mathrm{ppb}$ is good to use, but more than this rate it becomes dangerous. Although, even low levels of lead in the blood of children can result in behavior and learning problems, Lower IQ and hyperactivity, slowed growth, hearing problems, and anaemia studying results.

$$
\begin{gathered}
A=\sigma * \operatorname{randn}(\mathrm{n}, 1)+\lambda, \\
\mathrm{w}=\operatorname{wblrnd}(\sigma, \lambda,[\mathrm{n}, 1]),
\end{gathered}
$$

where $\sigma$ is a standard deviation; $\lambda$ is mean; $\lambda^{\prime}$ is a guess lambda. 

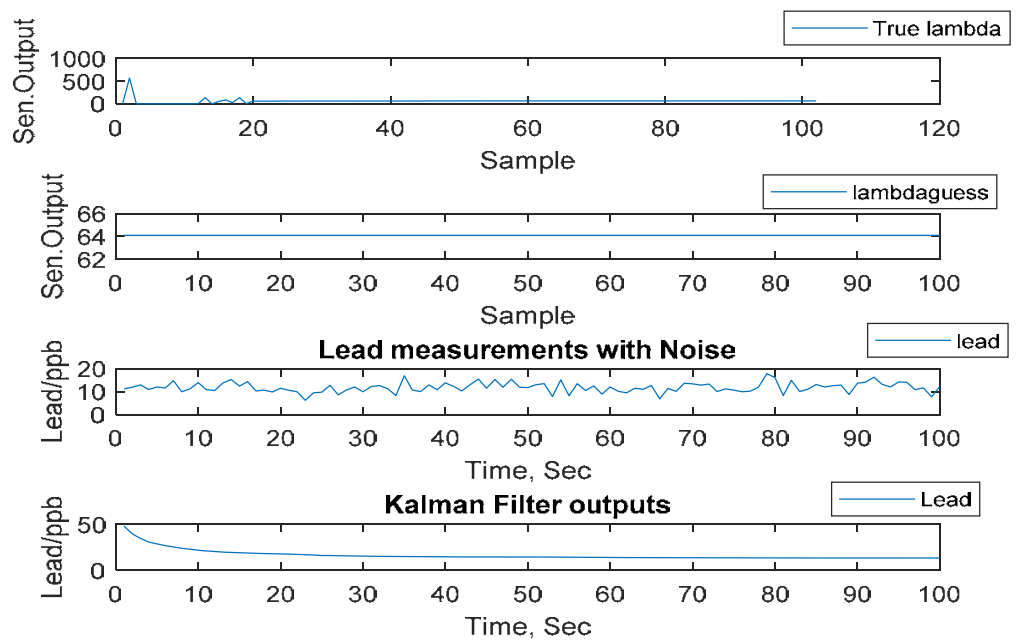

(a)
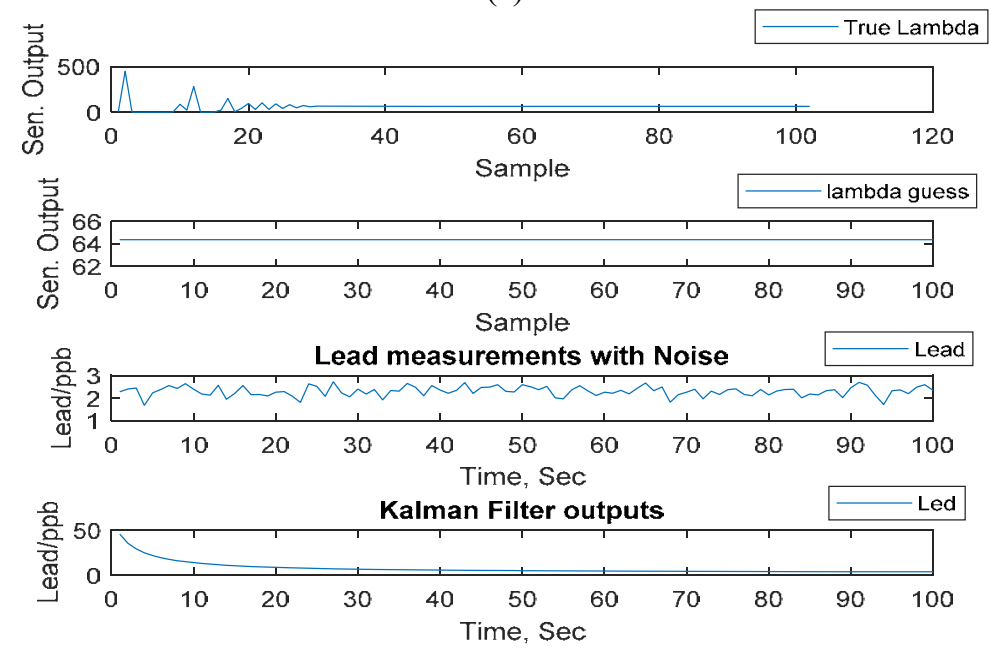

(b)

Figure 3: Measurements of lead rate on the water for (a) Weibull; (b) Uniform.

Table 1: Study results of lead.

\begin{tabular}{|l|c|c|c|c|c|c|c|}
\hline Real data & $\lambda$ & $\lambda^{\prime}$ & Gaussian & $\lambda^{\prime}$ & Weibull & $\lambda^{\prime}$ & Uniform \\
\hline $3 \mathrm{ppb}$ & 10 & 10.007 & $2.73 \mathrm{ppb}$ & 10.0181 & $2.795 \mathrm{ppb}$ & 10.02 & $2.81 \mathrm{ppb}$ \\
\hline $4 \mathrm{ppb}$ & 5 & 5.0738 & $3.99 \mathrm{ppb}$ & 5.0737 & $4.002 \mathrm{ppb}$ & 5.0697 & $3.99 \mathrm{ppb}$ \\
\hline $16 \mathrm{ppb}$ & 20 & 20.024 & $16.05 \mathrm{ppb}$ & 20.020 & $16.97 \mathrm{ppb}$ & 20.03 & $16.06 \mathrm{ppb}$ \\
\hline $17 \mathrm{ppb}$ & 1 & 0.379 & $16.98 \mathrm{ppb}$ & 0.38 & $16.97 \mathrm{ppb}$ & 0.365 & $16.98 \mathrm{ppb}$ \\
\hline $65 \mathrm{ppb}$ & 25 & 24.90 & $64.78 \mathrm{ppb}$ & 24.94 & $64.8 \mathrm{ppb}$ & 24.94 & $64.85 \mathrm{ppb}$ \\
\hline
\end{tabular}




\subsection{Linear Kalman filter (LKF)}

Kalman filter is an iterative mathematical process that uses a set of equations and consecutive data input to a quickly estimate the true value, position, velocity, etc., of the object being measured, when the measured value contains unpredicted or random error, uncertainty, or variation and this estimation could be based on linear or non-linear systems [14]. Each kind requires different approach of Kalman filter. This research, the linear Kalman filter has been chosen since the measurement mixed with noise. Therefore, the algorithm of linear Kalman filter is explained. The negative sign (-) indicates the a priori value, and the positive sign (+) indicates a posterior value [15] as shown in following eqn (3).

$$
X_{k}=A_{k-1}+W_{k},
$$

where $X_{k}$ refers to vector state of $(\mathrm{N} \mathrm{x} 1)$ at time $k$, while $A$ is a fixed matrix, and this refers to the transition state matrix of the process from the stat $K$ to $K+1$. Also, $W_{k}$ is represent the white noise ( $\mathrm{N} \times 1)$ for the process, and $k-1$ represents the previous measurements. the following eqn 4 , it is present the observation equation [16].

$$
Y_{k}=H_{k}+V_{k} \text {, }
$$

where $Y_{k}$ is represent actual measurement of $X(\mathrm{M} \mathrm{x} \mathrm{1),} \mathrm{while} \mathrm{H}$ observation connection matrix of the state and measurement vector $(\mathrm{M} \mathrm{x} \mathrm{N})$ and $V_{k}$ refers to white noise for the measurements. $\mathrm{Q}$ and $\mathrm{R}$ matrices are representing the covariance of noise and we can write it as follow in eqns (5) and (6)

$$
\begin{aligned}
& Q=E\left[\begin{array}{ll}
W_{k} & W_{k}^{T}
\end{array}\right], \\
& R=E\left[\begin{array}{ll}
V_{k} & V_{k}^{T}
\end{array}\right] .
\end{aligned}
$$

\section{CONCLUSION AND FUTURE WORKS}

In this research, we suggest measuring the pollution of water by using wireless sensor networks (WSNs). It's better than using conventional methods. Where this method requires less effort, money and gives more accurate results than laboratory results. It helps to make decisions in the shortest possible time to avoid the dangers of using polluted water. Stochastic approximation method is considered to calculate the collected sensors data and compare it with real data that was collected from Michigan.gov website. Simulation result showed that the monitoring system has successfully calculated the data and the result was too close to the real data. For future works, we will pay attention to:

- Study more recent sensors from alert labs.

- Collecting more data for same problem.

- Sensor fusion.

- Complementary and similarity.

\section{ACKNOWLEDGEMENTS}

It is a pleasure to express my deepest gratitude and appreciation towards my adviser Dr. Mohamed Zohdy for all his knowledge, guidance and constructive advice due to his demand for excellence.

Special thanks to wireless sensor network researchers team, Farag, Mariam, Waleed and Haythem for their help and constructive feedback. 


\section{REFERENCES}

[1] Adu-Manu, K.S. et al., Water quality monitoring using wireless sensor networks: Current trends and future research directions. ACM Transactions on Sensor Networks (TOSN), 13(1), p. 4, 2017.

[2] Holger, K. \& Willig, A., Protocols and Architectures for Wireless Sensor Networks, John Wiley \& Sons, 2007.

[3] Kumar, M. \& Puri, A., A review of permissible limits of drinking water. Indian Journal of Occupational and Environmental Medicine, 16(1), pp. 40, 2012.

[4] United States Environmental Protection Agency, Basic information about lead in drinking water. www.epa.gov/ground-water-and-drinking-water/basic-informationabout-lead-drinking-water. Accessed on: 18 Jan. 2017.

[5] Wisely, J. \& Erb, R., Chemical Testing Could Have Predicted Flint's Water Crisis, Detroit Free Press, 2015.

[6] Charles Stewart Mott Foundation, The Flint: A Good River with a Bad Reputation, 2015.

[7] Wikipedia, Flint water crisis. https://en.wikipedia.org/wiki/Flint_water_crisis \#Studies. Accessed on: 2 Dec. 2017.

[8] World Health Organization, www.who.int/mediacentre/factsheets/fs391/en/.

[9] Hayat, M.N. et al., Review of cluster-based energy routing protocols for WSNs. International Journal of Interdisciplinary Research Centre, 2016.

[10] Alnahash, N.A. \& Zohdy, M.A., Studying water quality monitoring system based on wireless sensor networks (WSNs). International Journal of Innovative Research in Computer and Communication Engineering, 5(8), 2017.

[11] www.mlive.com/entertainment/flint/index.ssf/2014/07/flint_river_watershed coalitio.html

[12] www.reddit.com/r/IAmA/comments/41vml6/iama_flint_michigan_native_and_report er who has/

[13] Flint Residential Testing Report - results collected 1 January 2017 through 19 December 2017. www.michigan.gov/documents/flintwater/Test_Results_Flint Sorted by_Address 578457 7.pdf. Accessed on: 1 Mar. 2017.

[14] Lagnf, F.M. \& Zohdy, M., 3D target detection and classification based on radar sensor fusion. ICIC Express Letters, 12(3), 2018.

[15] Grewal, M.S., Kalman filtering. International Encyclopedia of Statistical Science, Springer Berlin Heidelberg, pp. 705-708, 2011.

[16] Estimation of Tidal Current using Kalman Filter Finite Element Method with AIC, Ryosuke SUGA Chuo University Department of Civil Engineering Faculty. http://slideplayer.com/slide/8296077/. 